\title{
Controlling size, shape and homogeneity of embryoid bodies using poly(ethylene glycol) microwells $\uparrow$
}

\author{
Jeffrey M. Karp, $\uparrow^{* a b}$ Judy Yeh, $\$^{c}$ George Eng, $+^{* d}$ Junji Fukuda, ${ }^{d}$ James Blumling, ${ }^{d}$ Kahp-Yang Suh, ${ }^{e}$ \\ Jianjun Cheng, ${ }^{f}$ Alborz Mahdavi, ${ }^{d}$ Jeffrey Borenstein, ${ }^{g}$ Robert Langer*bcd and Ali Khademhosseini*ab
}

\author{
Received 3rd April 2007, Accepted 3rd April 2007 \\ First published as an Advance Article on the web 2nd May 2007 \\ DOI: $10.1039 / b 705085 m$
}

Directed differentiation of embryonic stem (ES) cells is useful for creating models of human disease and could potentially generate a wide array of functional cell types for therapeutic applications. Methods to differentiate ES cells often involve the formation of cell aggregates called embryoid bodies (EBs), which recapitulate early stages of embryonic development. EBs are typically made from suspension cultures, resulting in heterogeneous structures with a wide range of sizes and shapes, which may influence differentiation. Here, we use microfabricated cell-repellant poly(ethylene glycol) (PEG) wells as templates to initiate the formation of homogenous EBs. ES cell aggregates were formed with controlled sizes and shapes defined by the geometry of the microwells. EBs generated in this manner remained viable and maintained their size and shape within the microwells relative to their suspension counterparts. Intact EBs could be easily retrieved from the microwells with high viability ( $>95 \%$ ). These results suggest that the microwell technique could be a useful approach for in vitro studies involving ES cells and, more specifically, for initiating the differentiation of EBs of greater uniformity based on controlled microenvironments.

\section{Introduction}

Embryonic stem (ES) cells have generated great enthusiasm because of their ability to expand in culture and to differentiate into a variety of therapeutic cell types ${ }^{1,2}$ such as hepatocytes, ${ }^{3-5}$ pancreatic beta cells, ${ }^{6}$ cardiomyocytes, ${ }^{7-9}$ osteoblasts, ${ }^{10,11}$ endothelial cells, ${ }^{12}$ and neural cells. ${ }^{13-17}$ ES cells are also useful as models for human disease and development. ${ }^{18}$ One of the major challenges with ES cell research is to develop technologies to culture cells under controlled homogenous conditions.

Directing the differentiation of ES cells is believed to be largely regulated by various microenvironmental factors, such as soluble growth factors, matrix components, as well as cell-cell contact molecules. ${ }^{19,20}$ During development, a cell's position within spatially distinct microenvironments generated inside the embryo governs fate decisions in a tightly conserved manner. These events are recapitulated in vitro, where cells at

${ }^{a}$ Department of Medicine, Brigham and Women's Hospital, Harvard Medical School, Boston, MA 02115, USA.E-mail: alik@mit.edu

${ }^{b}$ Harvard-MIT Division of Health Science and Technology,

Massachusetts Institute of Technology, Cambridge, MA 02139, USA.

E-mail: alik@mit.edu; rlanger@mit.edu

${ }^{c}$ Department of Biological Engineering, Massachusetts Institute of Technology, Cambridge, MA 02139,USA.E-mail: rlanger@mit.edu

${ }^{d}$ Department of Chemical Engineering, Massachusetts Institute of

Technology, Cambridge, MA 02139,USA.E-mail: rlanger@mit.edu

${ }^{e}$ School of Mechanical and Aerospace Engineering, Seoul National

University, Seoul, Republic of Korea

${ }^{f}$ Department of Materials Science and Engineering, University of Illinois at Urbana-Champaign, Urbana, IL 61801, USA

${ }^{g}$ Draper Laboratory, Cambridge, MA 02139, USA

$\dagger$ This paper is part of a special issue 'Cell and Tissue Engineering in

Microsystems' with guest editors Sangeeta Bhatia (MIT) and

Christopher Chen (University of Pennsylvania).

* Denotes equal contribution. the periphery of differentiating ES cell aggregates tend to differentiate into primitive endoderm while the cells at the center of aggregates tend to differentiate into primitive ectoderm cells. ${ }^{21}$ The interaction of these cells can then induce further specification of cells within the aggregates. ${ }^{22}$ Therefore, in vitro microenvironmental factors, such as the position of cells relative to others within an aggregate and aggregate size, could influence their outcome.

Most protocols for inducing ES cell differentiation involve the removal of self-renewing factors and the formation of cell aggregates, called embryoid bodies (EBs), which initiate many developmental processes. EBs, which can generate cells from all germ layers, ${ }^{19}$ are generally formed through suspension ${ }^{23}$ or hanging drop ${ }^{24}$ methods. In suspension cultures, ES cells are placed on non-adhesive tissue culture dishes that allow cells to "clump together" in solution and form aggregates. Although suspension cultures are scalable, the dynamic environment increases the probability of collision and further aggregation ${ }^{25}$ and can result in a large size distribution. Recent methods have addressed some of these limitations through the use of a pitched blade turbine to create axial flow: however, ES proliferation, viability and aggregation are sensitive to hydrodynamic forces that result from elevated stirrer speeds. ${ }^{26}$ The hanging drop method is an alternative approach to forming EBs. In this method, cells are placed within liquid droplets on an inverted substrate. Although this method has the benefit of controlled EB size, changing media without disrupting the EBs poses a difficult challenge, and formation of large numbers of EBs is cumbersome, which makes this approach difficult to scale-up.

The development of new methods that can be used to homogenously form EBs and control their differentiation may be beneficial. These methods may also be useful for 
high-throughput drug screening or embryo toxicity studies. ${ }^{27}$ An improved method for controlling EB formation should: provide homogenous conditions and allow precise control of the initial number of cells, size and shape; be simple to perform and cost-efficient; permit the delivery of soluble and bound cues to the cells in a highly controlled manner; permit the recovery of the aggregates for further experimentation; have the potential for scalability; and be easily adaptable for highthroughput screening experiments.

To accommodate these criteria we investigated the potential of using micro-/nano-scale approaches, which represent an emerging field in controlling the cellular microenvironment. ${ }^{28-31}$ In these approaches, engineering can be applied to modify and control the cell's interactions with its immediate microenvironment. For example, micropatterning of substrates can be used to localize cells within a particular region on the substrate to allow high-throughput testing of materialES cell interactions or differentiation. ${ }^{28,29,32-34}$ Furthermore, modifications to the substrate shape have been shown to induce mesenchymal stem cell fate decisions. ${ }^{35}$

In this study, we used soft lithography ${ }^{34,36}$ to fabricate microwells without the use of complicated chemicals or expensive clean rooms. Microwells were fabricated from photocrosslinked poly(ethylene glycol) (PEG), a cell-repellant and inert material that prevents cell adhesion. ${ }^{37}$ Although microwells have been previously used to control the fabrication of uniform shaped cell aggregates from differentiated cell lines $^{32}$ and have also been used to control the interaction of ES cells with a second cell type, ${ }^{33}$ the ability to homogenously control differentiation using microwells has not been demonstrated. Given the great interest in the field in interfacing micro-scale technologies with ES cells, ${ }^{32,33,38-41}$ we believe it is critical to characterize the impact of micro-scale manipulation on such intricate cell types. Specifically, since ES cells have the potential to spontaneously and non-homogenously differentiate during culture manipulation, it is important to develop methods which can improve the homogeneity of culture conditions, a topic that is pertinent to the field but seldom discussed. Using cell repellant PEG microwells we show that it is possible to template ES cell aggregates to generate large numbers of isolated EBs, within an array, with controlled sizes and shapes, and that this method significantly reduces the heterogeneity of marker expression. Furthermore, as observed from marker expression from all 3 germ layers, microwell generation of uniform EB does not affect the capacity for multilineage differentiation.

\section{Materials and methods}

\section{Materials}

All tissue culture media and serum were purchased from Gibco-Invitrogen Corporation, cell lines were purchased from American Tissue Type Collection and all chemicals were purchased from Sigma-Aldrich Chemical Company, unless otherwise indicated. Polydimethylsiloxane (PDMS) elastomer composed of pre-polymer and curing agent was purchased from Essex Chemical (Sylgard 184). Calcein AM, ethidium homodimer and FITC-labeled BSA (FITC-BSA) were purchased from Molecular Probes (Eugene, OR). Mouse leukemia inhibitory factor (LIF) was purchased from Chemicon International, Inc. (Temecula, CA). MC-480, the antibody against SSEA-1, was purchased from Developmental Hybridoma Bank (Iowa City, IA). Phycoerythrin-conjugated goat anti-mouse IgM antibody was purchased from Jackson ImmunoResearch (West Grove, PA). Alpha-1-fetoprotein (AFP) was purchased from DakoCytomation (Carpinteria CA). Brachyury and Nestin antibodies were purchased from Santa Cruz Biotechnology (Santa Cruz, CA). Phycoerythrinconjugated goat anti-rabbit IgG antibody was purchased from Sigma.

\section{Cell culture}

All cells were manipulated under sterile tissue culture hoods and maintained in a 95\% air-5\% $\mathrm{CO}_{2}$ humidified incubator at $37{ }^{\circ} \mathrm{C}$. Murine ES cells (R1 strain) were maintained on gelatintreated dishes on a medium comprised of $15 \%$ ES-qualified FBS and $1000 \mathrm{U} \mathrm{ml}^{-1}$ mouse leukemia inhibitory factor (LIF) in DMEM knockout medium. ES cells were fed daily and passaged every 3 days at a subculture ratio of 1:4.

\section{PDMS mould fabrication}

PDMS moulds were fabricated by curing prepolymer (Sylgard 184, Essex Chemical) on silicon masters patterned with SU-8 photoresist. To cure the PDMS prepolymer, a mixture of 10:1 silicon elastomer and the curing agent was poured on the master and incubated at $70{ }^{\circ} \mathrm{C}$ for $2 \mathrm{~h}$. The PDMS mould was then peeled from the silicon wafer and cleaned with ethanol and acetone prior to use. The patterns on the masters had depressed features of various shapes, which resulted in corresponding PDMS replicas with protruding features.

\section{Formation of EBs within microwells}

PEG microwells were prepared as previously described ${ }^{37}$ (Fig. 1). To create surfaces capable of PEG attachment, glass slides were treated with trimethylsilyl methacrylate (TMSMA) for $5 \mathrm{~min}$ and cured at $100{ }^{\circ} \mathrm{C}$ for $10 \mathrm{~min}$ to enhance the

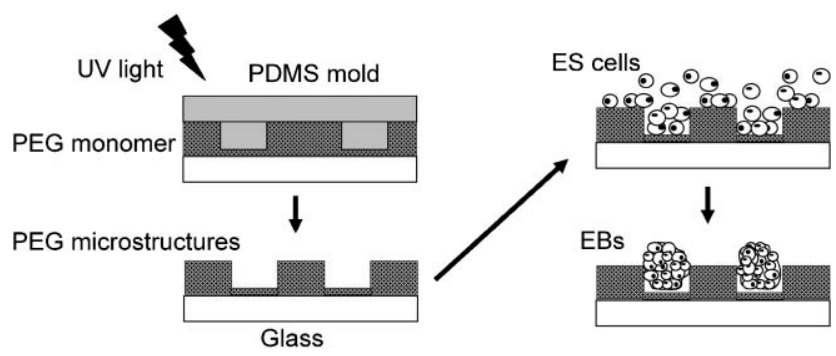

Fig. 1 Fabrication of PEG microwells and formation of an array of EBs. To fabricate PEG microwells, a PDMS mould containing protruding features was pressed onto a thick PEG polymer layer followed by crosslinking with UV and removal of the mould. Acrylate groups were used to anchor the photocrosslinkable PEG to the substrate. To generate EBs, a high density cell suspension was placed on the microwell arrays and allowed to settle within the wells. Subsequently, the array was gently washed to remove extraneous cells outside of the microwells, while cells within the microwells remained intact and shear-protected. The arrays were subsequently incubated and allowed to form EBs. 
adhesion of PEG to the surface. After rinsing $2 \times$ with DI water, a solution of $99.5 \mathrm{wt} \%$ PEGDA (MW 330) and $0.5 \mathrm{wt} \%$ of a water-soluble photoinitiator 2-hydroxy-2-methylpropiophenone photoinitiator was prepared. A few drops of the PEG polymer were evenly distributed onto the substrate, and a PDMS mould was then placed directly on the polymer film and exposed to $365 \mathrm{~nm}, 300 \mathrm{~mW} \mathrm{~cm}^{-2}$ UV light (EFOS Ultracure 100 ss Plus, UV spot lamp, Mississauga, Ontario) for $30 \mathrm{~s}$. To create EBs through immobilization of cells within the microwells, ES cells were trypsinized, re-suspended in medium at a concentration of $\sim 4 \times 10^{6}$ cells $\mathrm{mL}^{-1}$, plated on the PEG substrate in 6-well plates (with $\sim 5 \mathrm{ml}$ in each well), and allowed to settle overnight (Fig. 1). The cultures were then washed by a gentle flow of medium to remove cells that had not docked within the microwells. The cultures were returned to incubation at $37{ }^{\circ} \mathrm{C}$ and $5 \% \mathrm{CO}_{2}$ and fed with ES medium without LIF every 3-4 d. To harvest these EBs, a stream of medium is rinsed over the microwell surface using a P200 pipette. Each PEG microwell substrate is approximately $24.5 \mathrm{~mm} \times 24.5 \mathrm{~mm}$ in size (to fit within the wells in a 6-well plate), and the number of microwells per substrate ranges from $\sim 4000$ for the $150 \mu \mathrm{m}$ diameter microwells to $\sim 60000$ for the $40 \mu \mathrm{m}$ diameter microwells. The number of EBs used for each statistical analysis is specified in the legend for each graph. Depending on the initial seeding density relative to the microwell size, based on qualitative observation $\sim 75 \%$ of the microwells are completely filled with cells after an overnight incubation, and $\sim 50 \%$ cells initially seeded end up aggregating and completely filling the space within the microwells, and these are the cells that are used for the subsequent analyses.

\section{Cell viability and analysis}

To analyse cell viability, cells were incubated for $20 \mathrm{~min}$ with a solution containing ethidium homodimer and calcein AM dissolved at 4 and $2 \mu \mathrm{g} \mathrm{mL} \mathrm{mL}^{-1}$, respectively, in DMEM containing 10\% FBS. Live cells stained green while dead cells stained red, and they were visualized under an inverted fluorescent microscope (Axiovert 200, Zeiss).

\section{Cell differentiation and analysis}

ES cell self-renewal was analysed by fixing cells with 4\% paraformaldehyde for $10 \mathrm{~min}$ and staining cells with MC-480/SSEA-1 antibody (diluted 1:10 in a PBS solution) for $30 \mathrm{~min}$ and then phycoerythrin-conjugated goat anti-mouse IgM (diluted at 1:1000) for $30 \mathrm{~min}$. ES cell endodermal differentiation was analysed by fixing cells with $4 \%$ paraformaldehyde for $10 \mathrm{~min}$, permeabilizing cells with $0.2 \%$ Triton-X for $5 \mathrm{~min}$, and staining cells with AFP antibody (diluted 1:1000 in a PBS solution) for $30 \mathrm{~min}$ and then phycoerythrinconjugated goat anti-rabbit IgG (diluted at 1:20) for $30 \mathrm{~min}$. Appropriate controls were prepared by staining cells from each experimental group directly with the secondary antibody for SSEA-1 and AFP (without staining them with the primary antibody first). The fluorescence level of these control groups was then set as background and subtracted from that of the experimental groups. The fluorescence level was determined by using a flow cytometer (BD FACScans) and analysed using the
Cell Quest software. To provide a comparison against the cells grown in microwells of different sizes, ES cells from normal T-flask culture with LIF and EB cells formed from standard suspension culture without LIF were analyzed in parallel. Individual EBs were analysed by using the same staining protocol as described, with brachyury antibody (diluted at 1:200) and nestin antibody (diluted at 1:200), followed by phycoerythrin-conjugated rabbit anti-goat IgG (diluted at 1:20). Stained EBs were visualized and imaged using fluorescent microscopy, and the fluorescence of each EB was quantified using Scion Imaging and normalized with regard to the background fluorescence (of cells stained with secondary antibody only). To obtain fluorescent intensity values from individual EBs formed using microwells, the EBs were digitally isolated using the Scion image analysis program. The outer edge of microwells was ignored due to autofluorescence.

\section{Scanning electron microscopy}

To perform scanning electron microscopy (JEOL 6320FV), samples were mounted onto aluminium stages, sputter-coated with gold to a thickness of $200 \AA$ and analysed at a working distance of $20 \mathrm{~mm}$.

\section{Confocal microscopy}

For confocal microscopy, cells were stained with the membrane dye carboxyfluorescein diacetate, succinimidyl ester (CFSE) at $10 \mu \mathrm{g} \mathrm{ml}^{-1}$ in PBS, fixed with Fluoromount-G, and covered with a No. 1 thickness cover slip. Confocal images were taken at $10 \times$ magnification through a FITC filter with a maximum focal depth of $248 \mu \mathrm{m}$. CFSE was excited with an Argon laser at $488 \mathrm{~nm}$.

\section{Protein adsorption}

FITC-BSA was dissolved in PBS $(\mathrm{pH}=7.4)$ at $20 \mu \mathrm{g} \mathrm{mL}^{-1}$. To test for substrate exposure through protein adhesion, a few drops of the protein solution were evenly distributed onto the patterned substrates and incubated at room temperature for $45 \mathrm{~min}$. All patterned surfaces were then washed and analysed using an inverted fluorescent microscope (Axiovert 200, Zeiss).

\section{Results and discussion}

To develop a method of controlling EB formation with controlled sizes and shapes, a micro-scale template-based approach was used (Fig. 1). In addition, since cellular aggregates such as EBs are typically formed in suspension, surfaces that repel cells and proteins are desired. Microstructures were fabricated by micromoulding photocrosslinkable PEG, a cell-repellant hydrogel, as previously described. Using these features it was envisioned that the templating of ES cells within microfabricated non-adhesive microstructures could be used to generate EBs with controlled sizes and shapes. This system can easily be adapted to include other micro-scale techniques to localize cells within a particular region on the substrate or to perform highthroughput analysis of the effects of various conditions on ES cell differentiation. ${ }^{28,29,35}$ 

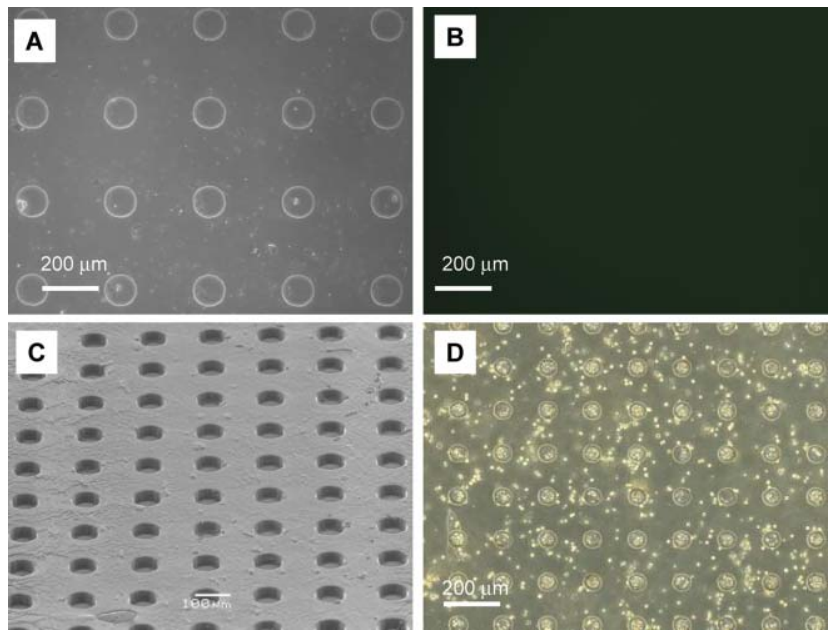

Fig. 2 Light (A) and fluorescent (B) images of PEG microwells that were treated with FITC-BSA. BSA adsorption to PEG-coated surfaces was undetectable using fluorescence imaging in contrast to glass or acrylate controls, where significant adsorption of protein was detected (not shown). Part (C) is an SEM image of PEG microwell patterns showing the high fidelity of the microwell array. Part (D) illustrates cell settling within microwells using a high seeding density.

(a)

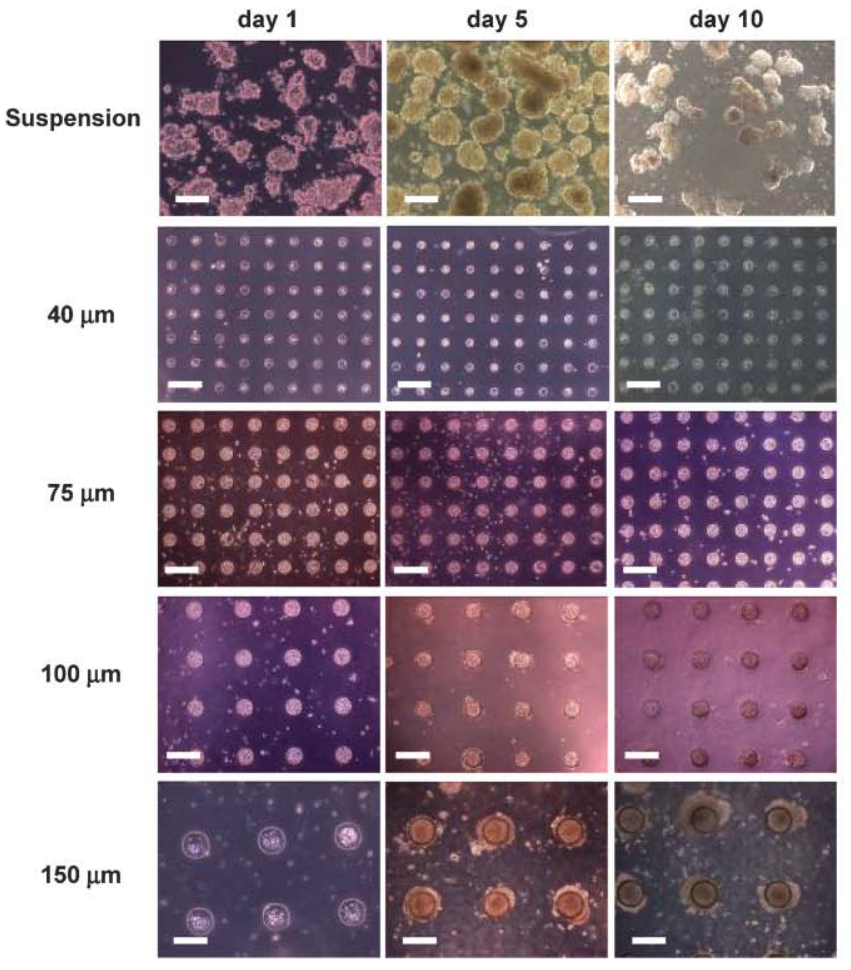

The cell-repellant properties of PEG microwells were evaluated by assessing their resistance to protein adsorption (Fig. 2). Specifically, PEG-coated surfaces resisted adhesion of FITC-BSA (Fig. 2A, B) in comparison with glass or acrylated controls, as observed by the lack of FITC staining compared with non-coated surfaces (not shown). This demonstrated that these microwells were fully coated with PEG and thus cells which were captured within these aggregates would not be exposed to protein-coated surfaces. Scanning electron microscopy (SEM) images of PEG microwells demonstrate that PEG microstructures can be fabricated with high-pattern fidelity (Fig. 2C). This facilitates the potential of scale-up without compromising the homogeneity of system, as is seen with suspension cultures where EBs may aggregate. ${ }^{25}$ As shown in Fig. 2D, cells successfully docked within the microwells. Furthermore, once the cells had settled within these regions they were not easily displaced, whereas excess cells could be easily rinsed away.

To evaluate the feasibility of PEG microwells for controlling the size of EBs, cells were confined within microwells with diameters ranging from $40 \mu \mathrm{m}$ to $150 \mu \mathrm{m}$ and heights of approximately $20 \mu \mathrm{m}$ to $35 \mu \mathrm{m}$ (Fig. 3(a)). The general procedure for generating such arrays involved plating a high
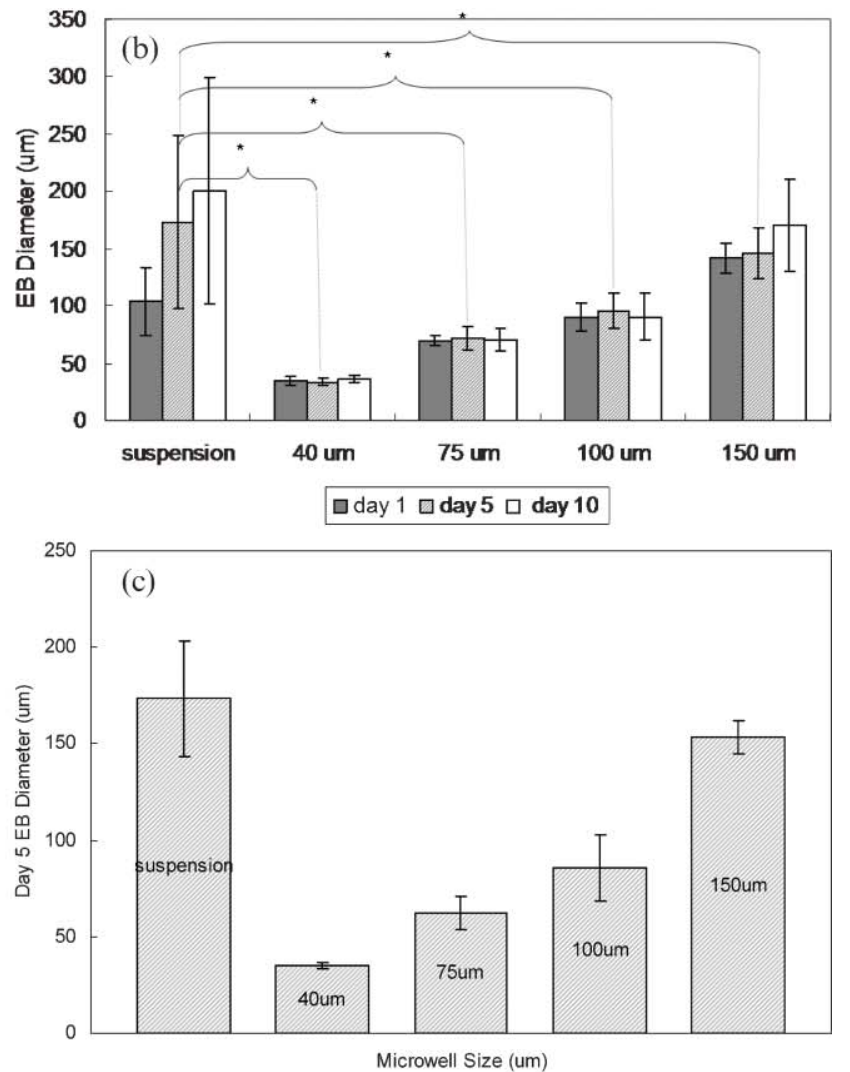

Fig. 3 (a) Time course of ES cells within PEG microstructures of different sizes. Each row is a specific microwell size (suspension culture, microwells with diameters of 40,75, 100 and $150 \mu \mathrm{m}$ ), and each column is at a different time point (day 1, 5 and 10). Scale bars correspond to $200 \mu \mathrm{m}$. (b) The size of ES cell aggregates grown without LIF in 40, 75, 100 and $150 \mu \mathrm{m}$ microwells was quantified on days 1, 5, and 10 and compared with suspension culture (without size control). Data are shown here as average \pm sd for $n=50$ samples within one experiment. (c) The size of ES cell aggregates grown without LIF in 40, 75, 100 and $150 \mu \mathrm{m}$ microwells after 5 days in culture was analysed across 3 independent experiments and compared with suspension culture (without size control). Data are shown here as average \pm sd for $n=3$ experiments. $*$ Indicates a statistically significant difference in variance $(F$ test value $<0.05)$. 
cell density $\left(\sim 4 \times 10^{6}\right.$ cells $\left.\mathrm{cm}^{-2}\right)$ on the PEG substrates and allowing the cells to settle within the microwells. Through seeding an excess number of cells, microwells of the same size can be filled with approximately the same number of cells. The presence of microwells creates regions of low shear stress, preventing the aggregates from being displaced during cell culture maintenance. Unlike suspension cultures, where the size of EBs may be highly variable and where control over the size of EBs may require an increase in propeller speed which may compromise cell viability, culturing of EBs within the microwells achieves highly defined dimensions with little variability (Fig. 3(a), (b)). Statistical analysis was performed using the $F$-test, which returns the result of the one-tailed probability that the variances in the two data sets compared are not significantly different. The results for day 5 (Fig. 3(a)) show that the differences in variance observed between groups are statistically significant $(p<0.05)$, thus verifying the relative homogeneity in size of EBs created through the microwell approach. When the time course diameter of the EBs was examined, it was found to be constant for over $10 \mathrm{~d}$ within all sizes of microwells with a small standard deviation compared with that of suspension culture EB controls (Fig. 3(b)). In addition to smaller variation within an experiment for the microwell technique, it was found that the variation in aggregate size was also smaller across multiple experiments (Fig. 3(c)).

Live/dead staining demonstrated that the cells within the EBs remained viable throughout a $10 \mathrm{~d}$ culture period (Fig. 4 A-D) irrespective of the microwell size. In addition to cultivating arrays of homogenous EBs of defined dimensions, a main advantage of using cell-repellant microwell templates is that EBs can be easily harvested from the wells at any time without compromising cell survival (Fig. 4 $\mathrm{E}-\mathrm{H})$. Overall, it is shown that EBs grown in microwells maintain their shape and a high degree of homogeneity in comparison with EBs that are generated using suspension cultures (Fig. 3). This permits the use of these EBs for future experimentation or potentially for therapeutic use. In addition, it is possible to interface EBs housed within microwells with microfluidics for high-throughput screening applications, ${ }^{42}$ after which the EBs can be recovered and further analyzed.

The EBs which were fabricated using this method were disk shaped. EBs created from suspension cultures are often spherical and typically quite heterogeneous in size (Fig. 3(a)). Interestingly, we found that the shape of the EBs did not significantly affect the expression of undifferentiated or differentiated markers after $5 \mathrm{~d}$. However, after $15 \mathrm{~d}$ trends in SSEA-1 and AFP expression indicate differences between microwell and suspension cultures, including differences among $50 \mu \mathrm{m}$ and $100 \mu \mathrm{m}$ microwells. Future work is required to determine the nature of these differences, which may be a function of EB size, shape, and aspect ratio. Importantly, the microwell system is well suited for interrogating the effect of these variables on ES cell differentiation. ${ }^{31}$ Specifically, microwells can be engineered with various aspect ratios and sizes. To examine parameters such as the surface area to volume ratio of the EBs and thus the ratio of cell-cell to cell-soluble environment interactions. In addition, the aggregate shape may affect mechanical forces transduced within the aggregate leading to differences in phenotype, as has recently been demonstrated. ${ }^{43}$

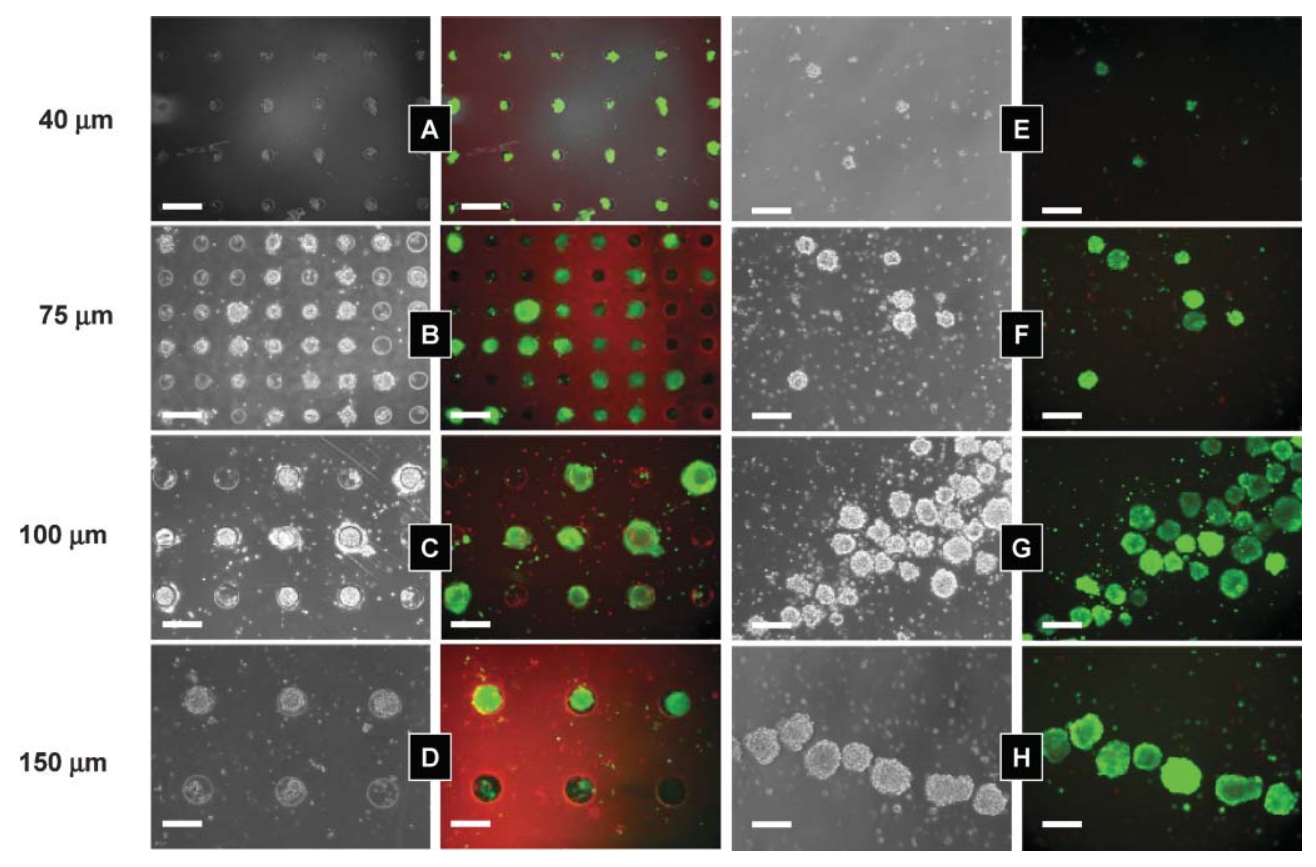

Fig. 4 Cells seeded in PEG microwells of different sizes: $40 \mu \mathrm{m}$ (A), $75 \mu \mathrm{m}$ (B), $100 \mu \mathrm{m}$ (C), and $150 \mu \mathrm{m}$ (D) were stained for viability after $10 \mathrm{~d}$ both within microwells and after cell aggregates were harvested from the microwells (E, F, G, and H, respectively). Viability was measured using Molecular Probes' live-dead stain, where live cells metabolize calcein AM and fluoresce green while dead cells uptake ethidium homodimer and fluoresce red. Columns 1 and 3 show light microscope images of cell aggregates whereas columns 2 and 4 show fluorescent images. Scale bars correspond to $200 \mu \mathrm{m}$. 


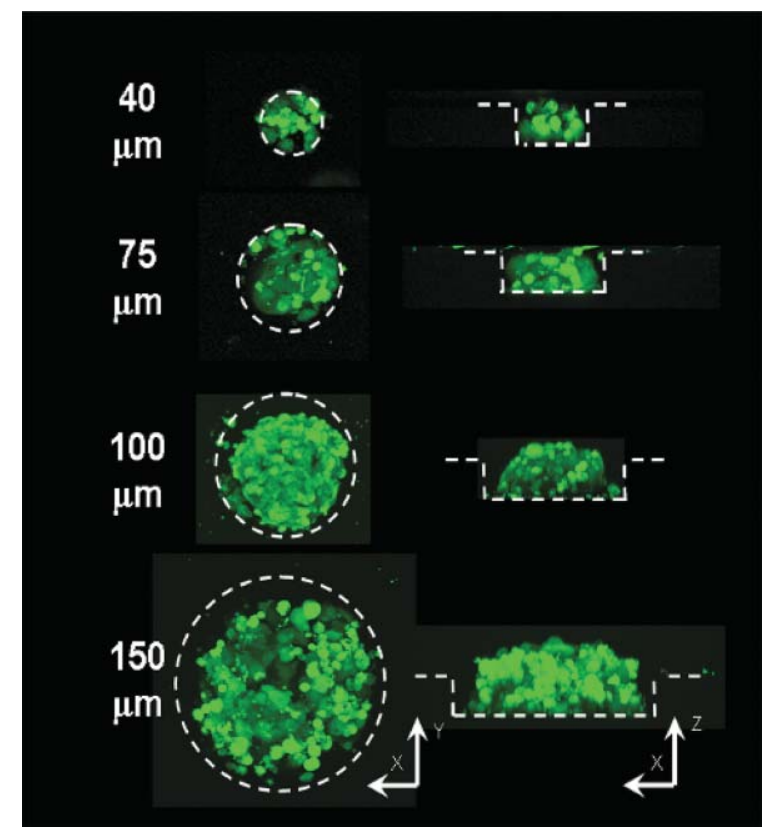

Fig. 5 Confocal laser light microscopy images of cell aggregates within $40 \mu \mathrm{m}, 75 \mu \mathrm{m}, 100 \mu \mathrm{m}$, and $150 \mu \mathrm{m}$ microwells 5 days after being stained with CFSE. Cells formed three-dimensional EB with diameters closely corresponding to those of their respective microwells. The first column of images shows aerial views of the cell aggregates whereas the second column shows vertical cross sections of cell aggregates within PEG microwells.

Confocal laser light microscopy (Fig. 5) demonstrated that ES cells conformed to the dimensions of the microwells regardless of microwell diameter $(40-150 \mu \mathrm{m})$. Although the diameter of the EBs grown within the microwells did not change significantly during the $10 \mathrm{~d}$ of culture (Fig. 3(b)), confocal images show that EBs formed in the large well sizes (100 and $150 \mu \mathrm{m}$ ) grew out of the microwells heightwise while maintaining the same diameter on the plane of the substrate surface. Therefore, cell-repellant microwells do not appear to inhibit cell growth. To prevent the cells from growing outside the microwells, the height can simply be controlled by changing the mould dimensions, the depth of compression (moulding pressure), or the viscosity of the moulded polymer.

In addition to modifying the diameter and height of the microwells, the shape can also be easily modified (Fig. 6). Previous reports have shown that the substrate shape can have a profound effect on cell function. ${ }^{44,45} \mathrm{We}$ demonstrate that uniform cell docking within microwells is not limited to cylindrical shapes (Fig. 6 B, E, F). Other geometries can be easily attained as well, given that the desired pattern is rendered in the original photomask design from which the corresponding silicon wafers and PDMS moulds are generated. Furthermore, even irregularly shaped EBs can be harvested from the microwells (Fig. 6C), making it possible to conduct further analysis for tissue engineering applications.

To examine the potential advantages of a microscale system that exhibits homogeneity in EB size and shape, we cultured ES cells within microwells in the absence of LIF, which represented differentiating conditions, and examined the expression of SSEA-1 and AFP markers for self-renewal and endoderm development, respectively (Fig. 7(a)). We compared these results to suspension cultures of EBs in the absence of LIF and to ES cells grown in the presence of LIF which served as controls. Results obtained for the suspension cultures were in agreement with previously reported mouse ES cell expression levels of SSEA-1 and AFP after 2 weeks in LIF conditions. ${ }^{46,47}$ Furthermore, results from antibody staining after $15 \mathrm{~d}$ of culture under differentiating conditions suggest that cells grown in microwells of controlled sizes may exhibit less variation in differentiation expression than those grown in standard suspension cultures without size control.

We believe that one of the unique features of the microwell approach of generating EBs is the ability to control the EB
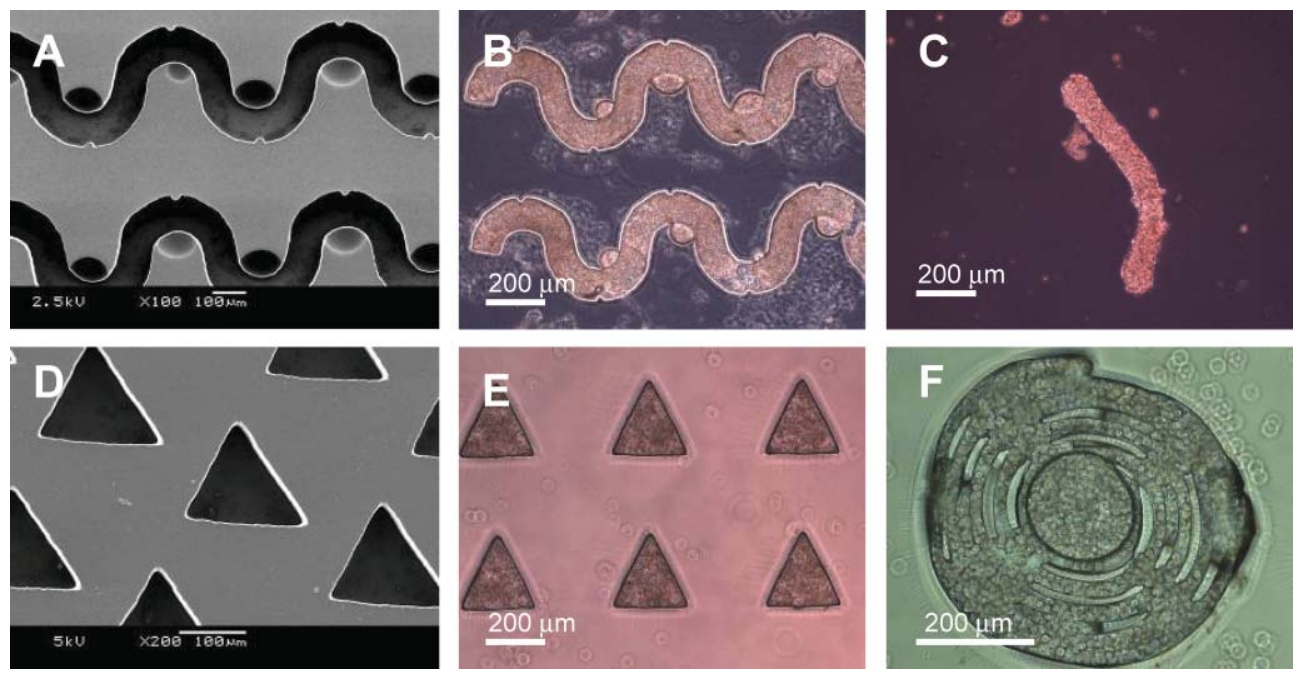

Fig. 6 PEG microstructures of different shapes: curves (A-C), triangles (D, E), and swirls (F), for example, were fabricated, imaged using SEM, and seeded with cells. When removed from the microstructures 3 or more days later, the cell aggregates are shown to maintain their shape, as previously moulded within the microwells (C). 

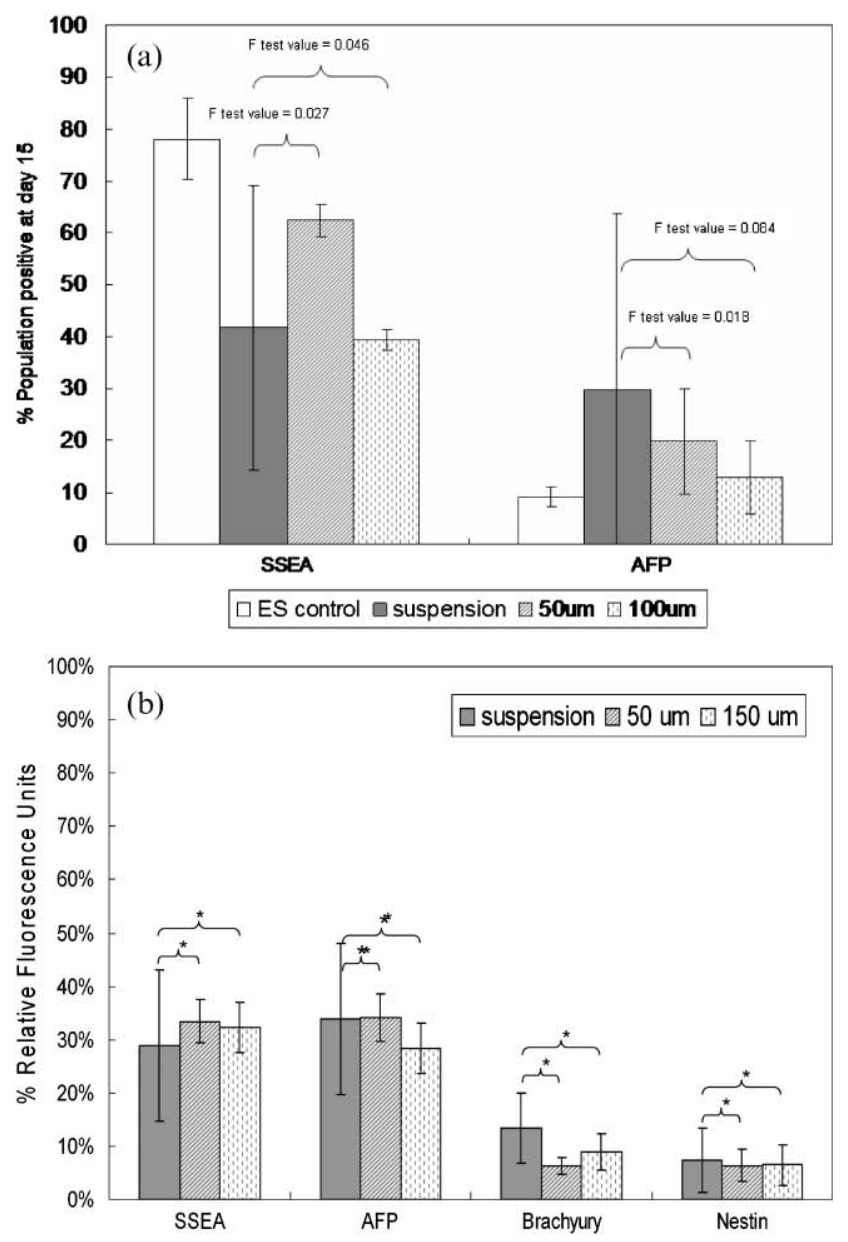

(c)

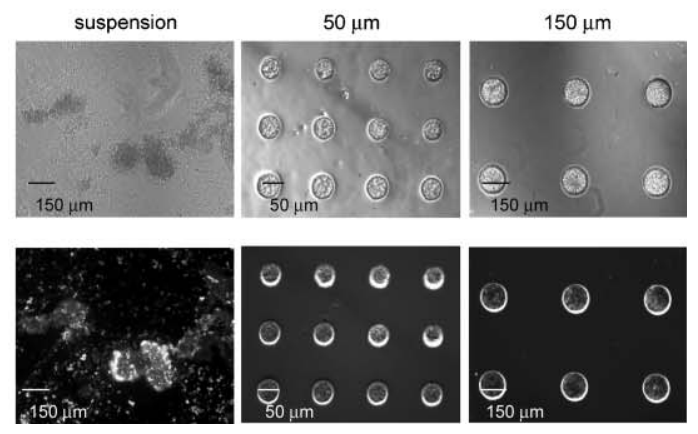

Fig. 7 (a) ES cells grown without LIF in 50 and $100 \mu \mathrm{m}$ microwells after $15 \mathrm{~d}$ in culture were analysed for SSEA-1 and AFP expression across 3 independent experiments (each with 10000 data points collected), in comparison with that of ES cells grown in normal T-flask culture with LIF and that of EB cells formed in standard suspension culture without LIF. Data are shown here as average \pm sd for $n=3$ experiments. (b) Quantitative single cell and (c) qualitative whole EB analysis of SSEA-1 (self-renewal), AFP (endoderm), Brachyury (mesoderm), and Nestin (ectoderm) expression in EBs (grown without LIF) in suspension, 50 and $100 \mu \mathrm{m}$ microwells after $5 \mathrm{~d}$. Cultures were analysed using immunostaining techniques and fluorescence microscopy. Data in (b) are shown as average \pm sd for $n=20$ individual EBs. * Indicates a statistically significant difference in variance $(F$ test value $<0.05)$.

variability. In addition to examining the homogeneity of SSEA-1 and AFP expression in individual cells after $15 \mathrm{~d}$ (Fig. 7(a)), we have performed immunostaining of SSEA-1 (self-renewal), AFP (endoderm), Brachyury (mesoderm), and Nestin (ectoderm) on individual EBs without LIF after $5 \mathrm{~d}$ to examine the homogeneity of differentiation within individual EBs (Fig. 7 (b), (c)). To quantify these differences we have calculated and compared the fluorescence intensity of individual EBs for each stain. The variation in expression of EBs grown in microwells was shown to be significantly smaller $(F$-test value $<0.05)$ than that of EBs grown in suspension. These results indicate that the variability of the differentiation between the EBs is smaller between EBs within the microwells than that of the EBs in the suspension cultures. Together, these results demonstrate that microwell cultures are useful for obtaining a greater homogeneity in differentiation pattern, both in terms of individual cells and in terms of individual EBs, thus minimizing the heterogeneity of differentiation that is typically observed with suspension cultures. This potential improvement in homogeneity may be beneficial in reproducibly differentiating stem cells into a specific lineage for potential tissue engineering and organ regeneration applications. In light of these encouraging preliminary results, it is worth pursuing further links between EB geometry and differentiation characteristics.

\section{Conclusion}

Here we show that homogenous and controllable EBs can be formed within microfabricated cell-repellant PEG wells which were used as a template to initiate the EB formation process. The size and shape of the EBs could be controlled, and the EBs formed in this manner remained viable and could be easily harvested from the wells. In addition, EBs in the microwells maintained their size and shape for at least $10 \mathrm{~d}$. Most importantly, this technique can be used for homogenously initiating the formation of EBs. Based on these approaches, it is possible to create arrays of EBs which might potentially be used (along with other microenvironmental factors) to direct ES cell differentiation by controlling the early stages of differentiation. The technique presented here can be easily incorporated into high-throughput systems, where generation of large numbers of homogenous EBs may be required for therapeutic use or for simultaneously studying the effects of multiple conditions on ES cell differentiation. This will be beneficial for a variety of applications that involve stem cells, such as tissue engineering, investigational cell biology, drug discovery, and high-throughput screening. In addition, this approach can be used in any laboratory equipped for cell culture, therefore making it a practical method for many biological applications.

\section{Acknowledgements}

This research has been supported by NIH (NIH grant \# HL60435), Draper laboratory, Institute of Soldier Nanotechnology (DAAD-19-02-D-002), and the NSF (through the Bioprocess Engineering Research Center). JF is supported by a Grant-in-Aid for JSPS fellows, 16-4754, 2004.

\section{References}

1 J. A. Thomson, J. Itskovitz-Eldor, S. S. Shapiro, M. A. Waknitz, J. J. Swiergiel, V. S. Marshall and J. M. Jones, Embryonic stem 
cell lines derived from human blastocysts, Science, 1998, 282(5391), $1145-1147$.

2 F. M. Watt and B. L. Hogan, Out of Eden: stem cells and their niches, Science, 2000, 287(5457), 1427-1430.

3 T. Hamazaki, Y. Iiboshi, M Oka, P. J. Papst, A. M. Meacham, L. I. Zon and N. Terada, Hepatic maturation in differentiating embryonic stem cells in vitro, FEBS Lett., 2001, 497(1), 15-19.

4 T. Ishii, K. Yasuchika and H. Fujii, In vitro differentiation and maturation of mouse embryonic stem cells into hepatocytes, Exp. Cell Res., 2005, 309(1), 68-77.

5 N. Lavon, O. Yanuka and N. Benvenisty, Differentiation and isolation of hepatic-like cells from human embryonic stem cells, Differentiation, 2004, 72(5), 230-238.

6 Y. Moritoh, E. Yamato, Y. Yasui, S. Miyazaki and J. Miyazaki, Analysis of insulin-producing cells during in vitro differentiation from feeder-free embryonic stem cells, Diabetes, May 2003, 52(5), $1163-1168$

7 C. Mummery, D. Ward-van Oostwaard and P. Doevendans, Differentiation of human embryonic stem cells to cardiomyocytes: role of coculture with visceral endoderm-like cells, Circulation, 2003, 107(21), 2733-2740

8 I. Kehat, A. Gepstein, A. Spira, J. Itskovitz-Eldor and L. Gepstein, High-resolution electrophysiological assessment of human embryonic stem cell-derived cardiomyocytes: a novel in vitro model for the study of conduction, Circ. Res., 2002, 91(8), 659-661.

9 I. Kehat, D. Kenyagin-Karsenti and M. Snir, Human embryonic stem cells can differentiate into myocytes with structural and functional properties of cardiomyocytes, J. Clin. Invest., 2001, 108(3), 407-414.

10 J. M. Karp, L. S. Ferreira, A. Khademhosseini, A. H. Kwon, J. Yeh and R. Langer, Cultivation of human embryonic stem cells without the embryoid body step enhances osteogenesis in vitro, Stem Cells, 2005 , in the press.

11 R. C. Bielby, A. R. Boccaccini, J. M. Polak and L. D. Buttery, In vitro differentiation and in vivo mineralization of osteogenic cells derived from human embryonic stem cells, Tissue Eng., 2004, 10(9-10), 1518-1525.

12 S. Levenberg, J. S. Golub, M. Amit, J. Itskovitz-Eldor and R. Langer, Endothelial cells derived from human embryonic stem cells, Proc. Natl. Acad. Sci. U. S. A., 2002, 99(7), 4391-4396.

13 J. H. Kim, J. M. Auerbach and J. A. Rodriguez-Gomez, Dopamine neurons derived from embryonic stem cells function in an animal model of Parkinson's disease, Nature, 2002, 418(6893), 50-56.

14 L. M. Bjorklund, R. Sanchez-Pernaute and S. Chung, Embryonic stem cells develop into functional dopaminergic neurons after transplantation in a Parkinson rat model, Proc. Natl. Acad. Sci. U. S. A., 2002, 99(4), 2344-2349.

15 S. C. Zhang, M. Wernig, I. D. Duncan, O. Brustle and J. A. Thomson, In vitro differentiation of transplantable neural precursors from human embryonic stem cells, Nat. Biotechnol., 2001, 19(12), 1129-1133.

16 M. Schuldiner, R. Eiges and A. Eden, Induced neuronal differentiation of human embryonic stem cells, Brain Res., 2001, 913(2), 201-205.

17 B. E. Reubinoff, P. Itsykson and T. Turetsky, Neural progenitors from human embryonic stem cells, Nat. Biotechnol., 2001, 19(12), $1134-1140$

18 J. J. Heit and S. K. Kim, Embryonic stem cells and islet replacement in diabetes mellitus, Pediatr. Diabetes, 2004, 5, $5-15$.

19 G. M. Keller, In vitro differentiation of embryonic stem cells, Curr. Opin. Cell Biol., 1995, 7(6), 862-869.

20 M. Schuldiner, O. Yanuka, J. Itskovitz-Eldor, D. A. Melton and N. Benvenisty, Effects of eight growth factors on the differentiation of cells derived from human embryonic stem cells, Proc. Natl. Acad. Sci. U. S. A., 2000, 97(21), 11307-11312.

21 T. Hamazaki, M. Oka, S. Yamanaka and N. Terada, Aggregation of embryonic stem cells induces Nanog repression and primitive endoderm differentiation, J. Cell Sci., 2004, 117(23), $5681-5686$

22 S. Pompe, M. Bader and C. Tannert, Stem-cell research: the state of the art. Future regulations of embryonic-stem-cell research will be influenced more by economic interests and cultural history than by ethical concerns, EMBO Rep., 2005, 6(4), 297-300

23 J. Itskovitz-Eldor, M. Schuldiner and D. Karsenti, Differentiation of human embryonic stem cells into embryoid bodies compromising the three embryonic germ layers, Mol. Med., 2000, 6(2), 88-95.

24 J. S. Boo, Y. Yamada and Y. Okazaki, Tissue-engineered bone using mesenchymal stem cells and a biodegradable scaffold, J. Craniofacial Surg., 2002, 13(2), 231-239, discussion 240-233.

25 S. M. Dang, M. Kyba, R. Perlingeiro, G. Q. Daley and P. W. Zandstra, Efficiency of embryoid body formation and hematopoietic development from embryonic stem cells in different culture systems, Biotechnol. Bioeng., 2002, 78(4), 442-453.

26 M. Schroeder, S. Niebruegge and A. Werner, Differentiation and Lineage Selection of Mouse Embryonic Stem Cells in a Stirred Bench Scale Bioreactor with Automated Process Control, Biotechnol. Bioeng., 2005, 92(7), 920-933.

27 A. Seiler, A. Visan, R. Buesen, E. Genschow and H. Spielmann, Improvement of an in vitro stem cell assay for developmental toxicity: the use of molecular endpoints in the embryonic stem cell test, Reprod. Toxicol., 2004, 18(2), 231-240.

28 D. G. Anderson, S. Levenberg and R. Langer, Nanoliter-scale synthesis of arrayed biomaterials and application to human embryonic stem cells, Nat. Biotechnol., 2004, 22(7), 863-866.

29 C. J. Flaim, S. Chien and S. N. Bhatia, An extracellular matrix microarray for probing cellular differentiation, Nat. Methods, 2005, 2(2), 119-125.

30 S. N. Bhatia, Customizing cellular microenvironments for hepatic tissue engineering, Abstr. Pap. Am. Chem. Soc., 2001, 221, U127-U127.

31 A. Khademhosseini, R. Langer, J. Borenstein and J. P. Vacanti, Microscale technologies for tissue engineering and biology, Proc. Natl. Acad. Sci. U. S. A., 2006, 103(8), 2480-2487.

32 J. Fukuda, A. Khademhosseini and Y. Yeo, Micromolding of photocrosslinkable chitosan hydrogel for spheroid microarray and co-cultures, Biomaterials, 2006, 27(30), 5259-5267.

33 A. Khademhosseini, L. Ferreira and J. Blumling, Co-culture of human embryonic stem cells with murine embryonic fibroblasts on microwell-patterned substrates, Biomaterials, 2006, 27(36), 5968-5977.

34 A. Revzin, K. Sekine, A. Sin, R. G. Tompkins and M. Toner, Development of a microfabricated cytometry platform for characterization and sorting of individual leukocytes, Lab Chip, 2005, 5(1), 30-37.

35 R. McBeath, D. M. Pirone, C. M. Nelson, K. Bhadriraju and C. S. Chen, Cell shape, cytoskeletal tension, and RhoA regulate stem cell lineage commitment, Dev. Cell, 2004, 6(4), 483-495.

36 Y. S. Kim, K. Y. Suh and H. H. Lee, Fabrication of threedimensional microstructures by soft molding, Appl. Phys. Lett., 2001, 79(14), 2285-2287.

37 A. Khademhosseini, J. Yeh and S. Jon, Molded polyethylene glycol microstructures for capturing cells within microfluidic channels, Lab Chip, Oct 2004, 4(5), 425-430.

38 J. C. Mohr, J. J. de Pablo and S. P. Palecek, 3-D microwell culture of human embryonic stem cells, Biomaterials, 2006, 27(36), 6032-6042.

39 P. U. Rani and J. S. Khillan, A simple and convenient method for preparing chimeric animals from embryonic stem (ES) cells, Transgenic Res., 2003, 12(6), 739-741.

40 D. R. Albrecht, G. H. Underhill, T. B. Wassermann, R. L. Sah and S. N. Bhatia, Probing the role of multicellular organization in three-dimensional microenvironments, Nat. Methods, 2006, 3(5), 369-375.

41 C. J. Flaim, S. Chien and S. N. Bhatia, An extracellular matrix microarray for probing cellular differentiation, Nat. Methods, $2005,2(2), 119-125$

42 A. Khademhosseini, J. Yeh and G. Eng, Cell docking inside microwells within reversibly sealed microfluidic channels for fabricating multiphenotype cell arrays, Lab Chip, 2005, 5(12), $1380-1386$.

43 C. M. Nelson, R. P. Jean and J. L. Tan, Emergent patterns of growth controlled by multicellular form and mechanics, Proc. Natl. Acad. Sci. U. S. A., 2005, 102(33), 11594-11599.

44 C. S. Chen, J. L. Alonso, E. Ostuni, G. M. Whitesides and D. E. Ingber, Cell shape provides global control of focal 
adhesion assembly, Biochem. Biophys. Res. Commun., 2003, 307(2), 355-361.

45 C. S. Chen, M. Mrksich, S. Huang, G. M. Whitesides and D. E. Ingber, Micropatterned surfaces for control of cell shape, position, and function, Biotechnol. Prog., 1998, 14(3), $356-363$.
46 T. Ishii, K. Yasuchika and H. Fujii, In vitro differentiation and maturation of mouse embryonic stem cells into hepatocytes, Exp. Cell Res., 2005, 309(1), 68-77.

47 A. Toumadje, K. Kusumoto and A. Parton, Pluripotent differentiation in vitro of murine ES-D3 embryonic stem cells, In Vitro Cell Dev. Biol. Anim., 2003, 39(10), 449-453.

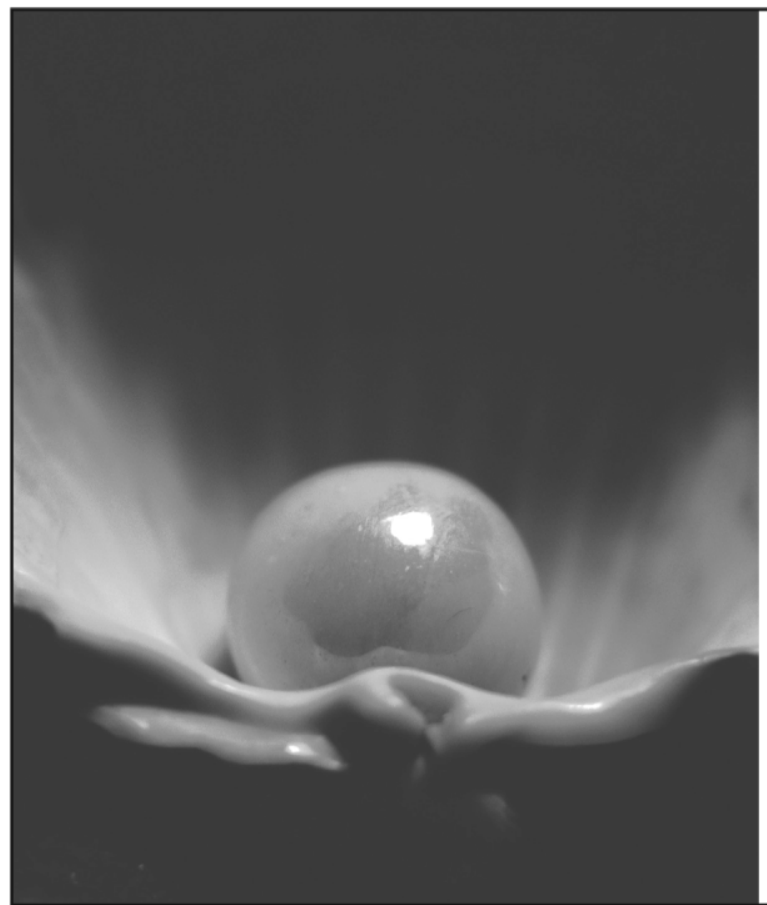

\section{Looking for that Special chemical science research paper?}

TRY this free news service:

\section{Chemical Science}

- highlights of newsworthy and significant advances in chemical science from across RSC journals

- free online access

- updated daily

- free access to the original research paper from every online article

- also available as a free print supplement in selected RSC journals.*

*A separately issued print subscription is also available.

Registered Charity Number: 207890 